\title{
Preparation of Conjugated Linoleic Acid Microemulsions and their Biodistribution
}

\author{
Kazuaki Kishita ${ }^{1}$, Kayo Ibaraki ${ }^{2}$, Shoko Itakura², Yumi Yamasaki ${ }^{3}$, Naoko Nishikata ${ }^{4}$, \\ Kenji Yamamoto ${ }^{4}$, Masataka Shimizu ${ }^{4}$, Kazuo Nishiyama ${ }^{1,2}$ and Masao Yamasaki ${ }^{1,2 *}$ \\ ${ }^{1}$ Interdisciplinary Graduate School of Agriculture and Engineering, University of Miyazaki, 1-1 Gakuenkibanadai-nishi Miyazaki 889-2192, JAPAN \\ ${ }^{2}$ Faculty of Agriculture, University of Miyazaki, 1-1 Gakuenkibanadai-nishi Miyazaki 889-2192, JAPAN \\ ${ }^{3}$ Faculty of Regional Innovation, University of Miyazaki, 1-1 Gakuenkibanadai-nishi Miyazaki 889-2192, JAPAN \\ ${ }^{4}$ Miyazaki Prefecture Industrial Technology Center, 16500-2 Higashi-Kaminaka, Sadawara-cho, Miyazaki 880-0303, JAPAN
}

\begin{abstract}
Conjugated linoleic acid (CLA) has several beneficial biological properties. Specifically, trans10, cis12-CLA, one of the CLA isomers, has strong physiologic activity against cancer and obesity. However, compared with cis9, trans11-CLA, a naturally occurring CLA isomer, trans10, cis12-CLA tends to be easily metabolized. Therefore, to make efficient use of its biological properties, it is necessary to overcome the rapid clearance of trans10, cis12-CLA from the blood. Here, we employed premix membrane emulsification to prepare two oil-in-water CLA microemulsions (CLA-ME), $100 \mathrm{~nm}$ CLA-ME and $200 \mathrm{~nm}$ CLA-ME, and investigated their pharmacokinetics in a mouse model. We report that $100 \mathrm{~nm}$ CLA-ME contributed to the concentration of blood CLA for longer than $200 \mathrm{~nm}$ CLA-ME, indicating that small CLA microparticles were more suitable for maintaining blood trans10, cis12-CLA levels in vivo. However, both CLA-ME could be hardly detected in blood and other tissues $24 \mathrm{~h}$ after administration, suggesting that additional strategies for prolonging CLA-ME half-life are required.
\end{abstract}

Key words: conjugated linoleic acid, microemulsions, premix membrane emulsification, biodistribution

\section{Introduction}

Conjugated linoleic acid (CLA) is a general term describing isomers of linoleic acid with conjugated double bonds. They are found mainly in milk, dairy products, and meat from ruminants, in which cis 9 , trans11-CLA $(c 9, t 11$-CLA) is the main isomer ${ }^{1)}$. Trans10, cis12-CLA $(t 10, c 12$-CLA $)$ is produced under strong alkaline conditions during hydrogenation of linoleic acid. Most studies have focused on the two main isomers: $c 9, t 11$-CLA and $t 10, c 12$-CLA, and reported their numerous beneficial biological effects, such as preventing tumors and atherosclerosis, or reducing obesity and hypertension ${ }^{2-6)}$. In particular, $t 10, c 12$-CLA appears to be effective against some cancer cell lines and obesity. For instance, we previously reported that $t 10, c 12$-CLA showed potent cytotoxic effect on liver cancer cells in vitro $^{7,8)}$. However, $t 10, c 12$-CLA tends to be rapidly metabolized and thus cleared from the blood stream ${ }^{9,10)}$. Therefore, oral administration of $t 10, c 12$-CLA failed to prevent allograft liver tumor in Donryu rats because this isomer did not accumulate in the cancer cells ${ }^{11)}$. Hence, a novel method for prolonging the biological function of CLA is re- quired. For its anti-cancer effect, we tried to develop a new strategy in which $t 10, c 12$-CLA is able to accumulate in cancer cells by microemulsification. Since vascular permeability in tumor tissues is highly increased, microparticles can pass through the blood walls and are provided access to the cancer cells ${ }^{12,13)}$. In this context, protection of the microparticle from phagocytotic processes and its stability in the blood stream are required under steady-state(nontumor) conditions. A previous report showed that oral administration of CLA-ME improved the anti-obesity effect of CLA by promoting CLA absorption ${ }^{14)}$. Although utilization of CLA-ME for oral administration has been investigated, tissue biodistribution and blood concentration of CLA-ME following intravenous injection remain unclear. In the present study, we prepared two sizes of CLA-ME by premix membrane emulsification, and evaluated their pharmacokinetic properties after intravenous administration in a mouse model.

\footnotetext{
${ }^{*}$ Correspondence to: Masao Yamasaki, Department of Biochemistry and Applied Biosciences, Faculty of Agriculture, University of Miyazaki 1-1 Gakuenkibanadai-nishi Miyazaki 889-2192, JAPAN

E-mail: myamasaki@cc.miyazaki-u.ac.jp

Accepted July 4, 2016 (received for review May 9, 2016)

Journal of Oleo Science ISSN 1345-8957 print / ISSN 1347-3352 online

http://www.jstage.jst.go.jp/browse/jos/ http://mc.manusriptcentral.com/jjocs
} 


\section{Experimental}

\subsection{Materials}

CLA triglyceride was obtained from Nissin Oillio (Tokyo, Japan). Polyethylne glycol(PEG)-60 hydrogenated castor oil (HCO-60) for clinical use was purchased from Nikoh Chemicals (Tokyo, Japan) and sodium cholate was purchased from Wako (Osaka, Japan).

\subsection{Preparation and characterization of CLA-ME}

The CLA-ME was prepared according to a previously reported method $^{15)}$. To obtain crude CLA emulsions, $20 \mathrm{~mL}$ CLA triglyceride was dissolved in $180 \mathrm{~mL}$ outer water phase consisting of $10 \%$ potassium-free PBS, $1 \%$ HCO-60, and $10 \%$ sodium cholate, after which the mixture was stirred with a magnetic stirrer at $700 \mathrm{rpm}$ for $30 \mathrm{~min}$ at room temperature (HPS-100, As One, Osaka, Japan). Particle size of CLA emulsions was analyzed using a laser diffraction particle size analyzer(SALD-2000; Shimadzu, Kyoto, Japan) followed by premix membrane emulsification. Based on the obtained results, we chose the appropriate pore size of Shirasu porous glass or anodic aluminum oxide(Whatman Anodisc; GE Healthcare, Little Chalfont, UK) membranes for emulsification, and analyzed mean particle size using a dynamic light scattering particle size analyzer (ELS-8000DC; Otsuka Electronics, Tokyo, Japan). The procedure was repeated until mean particle sizes of 200 and 100 nm were obtained. Finally, CLA-ME was dialyzed using a biotech cellulose ester membrane in order to remove sodium cholate for 3 hours (Spectra/PorR Biotech Cellulose Ester (CE) Dialysis Membranes MWCO 100,000, Spectrum Laboratories, CA). After preparation of CLA-ME, mean particle size, polydispersity index, and zeta potential were measured by ELS-8000DC. Viscosities $(\mathrm{mPa} \cdot \mathrm{s})$ of CLA-ME were measured using tuning fork viscometer (SV-1A, A\&D, Tokyo, Japan).

\subsection{Intravenous administration test}

Eight-week-old male BALB/c mice were purchased from Charles River (Kanagawa, Japan) and fed pellet diet and given water ad libitum. The animal experiments were conducted in accordance with the Guide for the Care and Use of Laboratory Animals at the University of Miyazaki and in compliance with the Law Concerning the Protection and Control of Animals (Japan Law No. 105), Standards Relating to the Care and Management of Laboratory Animals and Relief of Pain (Notification No. 88 of the Ministry of the Environment, Japan), and the Guidelines for
Animal Experimentation (Japanese Association for Laboratory Animal Science). Mice were maintained under 12-hour light/12-hour dark cycle at $20^{\circ} \mathrm{C}$, under conventional conditions. The mice were divided in three groups and injected $0.3 \mathrm{~mL}$ CLA-ME or outer water phase (as a control) via the tail vain at 9 a.m. At 1, 3, 6, and 24 h after administration, blood, heart, liver, spleen, kidney, and epididymal adipose tissues were collected. To obtain plasma and tissue homogenates, blood was removed by centrifugation at $800 \times g$ for $20 \mathrm{~min}$ at $4^{\circ} \mathrm{C}$, and the collected tissues were crashed using a bead beater-type homogenizer (MicroSmash MS-100R, Tomy, Tokyo, Japan).

\subsection{Gas chromatography (GC) analysis}

The plasma or homogenates were mixed with $1 \mathrm{~mL} 0.1$ $\mathrm{mg} / \mathrm{mL}$ aqueous tripentadecanoin and $3 \mathrm{~mL}$ chloroform/ methanol (2:1), and the mixture was centrifuged at $600 \times g$ for $5 \mathrm{~min}$. The lower layer was collected and the upper layer was mixed with $4 \mathrm{~mL}$ chloroform, followed by centrifugation at $600 \times g$ for $5 \mathrm{~min}$. The lower layer thus obtained was flushed with $\mathrm{N}_{2}$ gas in a $37^{\circ} \mathrm{C}$ water bath. After drying, oil extracts were mixed with $1 \mathrm{~mL} 0.5 \mathrm{M} \mathrm{KOH} /$ methanol and heated at $100^{\circ} \mathrm{C}$ for $5 \mathrm{~min}$. Next, the mixture was mixed with $0.4 \mathrm{~mL} 10 \% \mathrm{HCl} /$ methanol and heated at $100^{\circ} \mathrm{C}$ for 5 min. Finally, methylated fatty acids were extracted by hexane. CLA contents of plasma and other tissues were analyzed using a gas chromatograph (GC-14B; Shimadzu) . Gas chromatographic analysis was performed under the conditions described by a previous study ${ }^{16)}$.

\subsection{Statistical analysis}

Values in Table 1 are presented as mean $\pm \mathrm{SD}$, other results are presented as mean \pm SEM. Student's $t$-test was used to determine statistical significance between data at $p$ $<0.05(*)$ or $p<0.01(* *)$.

\section{Results}

CLA-ME was prepared by premix membrane emulsification and their characteristics were determined using a particle size/zeta potential analyzer. As shown in Table 1 and Fig. 1, we obtained ME of two sizes: $106.9 \pm 1.3 \mathrm{~nm}(100$ $\mathrm{nm}$ CLA-ME) and $191.8 \pm 1.7 \mathrm{~nm}(200 \mathrm{~nm}$ CLA-ME). The polydispersity index/zeta potential of $100 \mathrm{~nm}$ and $200 \mathrm{~nm}$ CLA-ME were $0.063 \pm 0.005 /-11.1 \pm 0.9 \mathrm{mV}$ and 0.037 $\pm 0.032 /-16.6 \pm 1.5 \mathrm{mV}$, respectively. There was no statisti-

Table 1 Characterization of CLA-ME.

\begin{tabular}{cccc}
\hline & PDI & Zeta potential $(\mathrm{mV})$ & Viscosity $(\mathrm{mPa} \cdot \mathrm{s})$ \\
\hline $100 \mathrm{~nm}$ CLA-ME & $0.063 \pm 0.005$ & $-11.1 \pm 0.9$ & $1.18 \pm 0.04$ \\
$200 \mathrm{~nm}$ CLA-ME & $0.037 \pm 0.032$ & $-16.6 \pm 1.5$ & $1.08 \pm 0.01$ \\
\hline
\end{tabular}

Data represent means \pm SD for three samples. PDI, polydispersity index. 

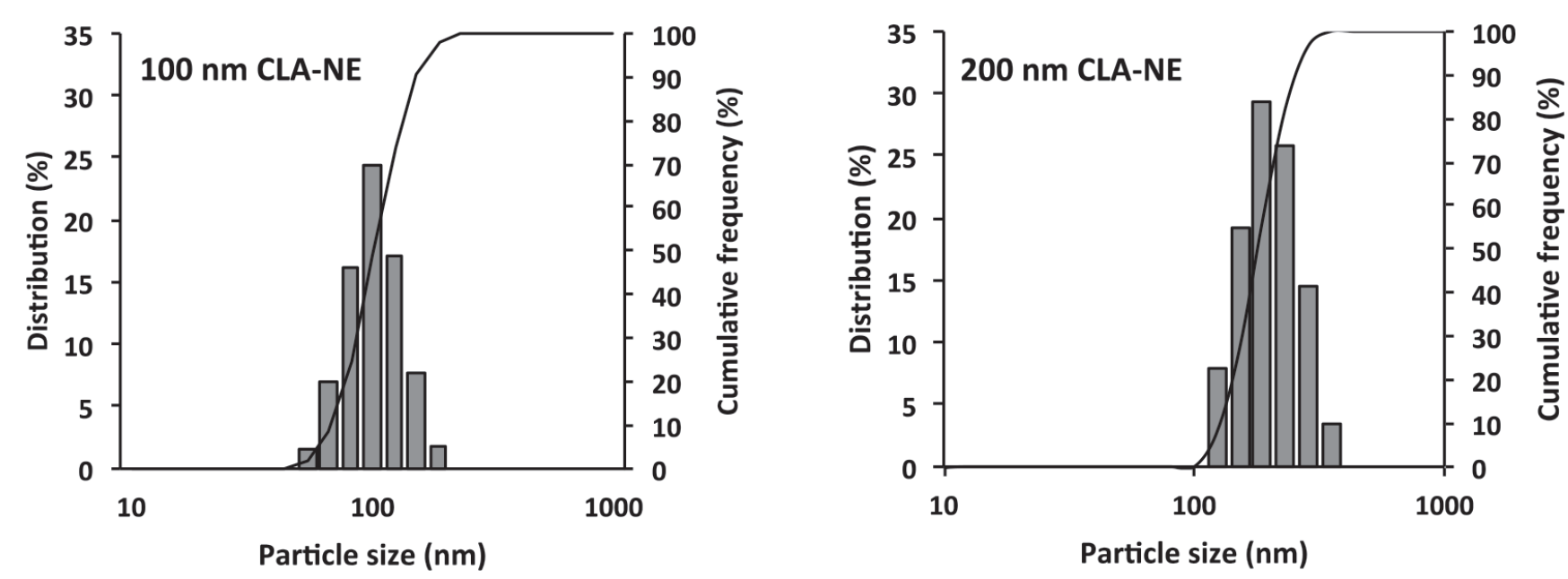

Fig. 1 Characteristics of CLA-ME. Particle size distribution (bar graphs) and cumulative frequency (line graphs) of CLA-ME.

Table 2 Fatty acid composition of CLA-ME.

\begin{tabular}{cccc}
\hline Fatty acids (wt.\% of total FA) & CLA-TG & $100 \mathrm{~nm}$ CLA-ME & 200 nm CLA-ME \\
\hline $16: 0$ & 3.6 & 4.0 & 3.8 \\
$18: 0$ & 2.4 & 2.3 & 1.8 \\
$18: 1$ & 11.6 & 11.4 & 11.7 \\
$18: 2(c 9, c 12)$ & 0.5 & 2.2 & 1.5 \\
\hline$C L A$ & & & \\
\hline$c 9, t 11$ & 35.8 & 34.9 & 38.7 \\
$t 10, c 12$ & 36.5 & 35.8 & 36.7 \\
$c 9, c 11 ; c 10, c 12$ & 1.4 & 1.5 & 1.8 \\
$t 9, t 11 ; t 10, t 12$ & 2.0 & 2.2 & 1.5 \\
Total CLA & 75.6 & 74.3 & 78.7 \\
\hline
\end{tabular}

CLA-TG; CLA triglyceride

cally significant difference in zeta potential between 100 $\mathrm{nm}$ and $200 \mathrm{~nm}$ CLA-ME. Fatty acid compositions of CLA-ME are shown in Table 2. Purity of CLA in $100 \mathrm{~nm}$ and $200 \mathrm{~nm}$ CLA-ME was $74.3 \mathrm{wt} \%$ and $78.8 \mathrm{wt} \%$ of total fatty acids, respectively.

To evaluate biodistribution of CLA-ME, we conducted an intravenous administration test in BALB/c mice. At 1, 3, 6, and $24 \mathrm{~h}$ after injection of CLA-ME, mice tissues were collected and analyzed for CLA contents by GC. After $6 \mathrm{~h}$, blood CLA concentration tended to be lower in mice treated with $200 \mathrm{~nm}$ CLA-ME than $100 \mathrm{~nm}$ CLA-ME ( $p$ values in each isomer $=0.08$ ), suggesting that the former tended to be cleared faster than the latter (Fig. 2). The half-life of each CLA-ME type was nevertheless similar $(7 \mathrm{~h}$ for $100 \mathrm{~nm}$ CLA-ME, $6 \mathrm{~h}$ for $200 \mathrm{~nm}$ CLA-ME). Both CLA-ME were detected in the liver, lung, kidney, heart and adipose tissue at $1 \mathrm{~h}$ post injection(Fig. 3). Although 200 nm CLA-ME were more abundant than $100 \mathrm{~nm}$ CLA-ME in liver tissue at $3 \mathrm{~h}$, that trend was reversed at $6 \mathrm{~h}$ but these differences were not significant. In addition, $200 \mathrm{~nm}$

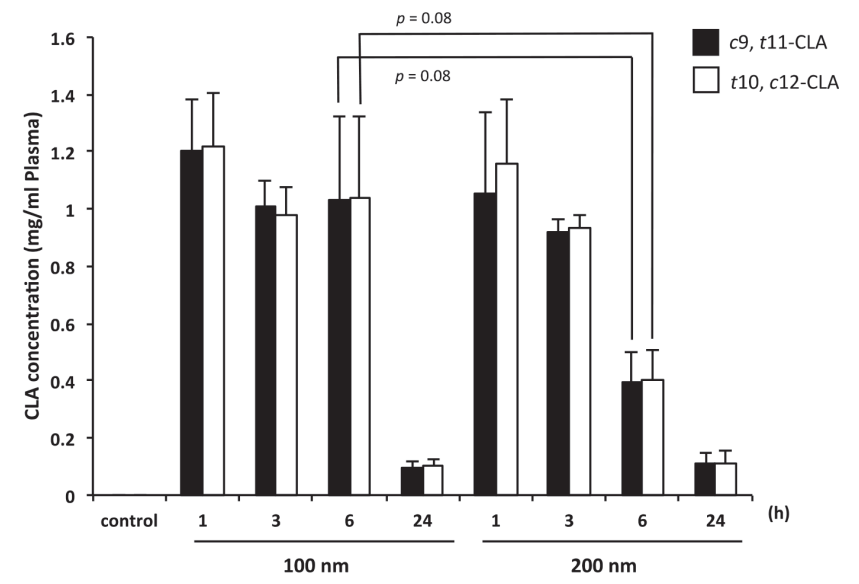

Fig. 2 CLA concentration in the blood of BALB/c mice. Mice were administered CLA-ME by intravenous injection, blood samples were collected at indicated times, and subjected to GC analysis. Data represent means \pm SEM for 3-4 mice per group. 

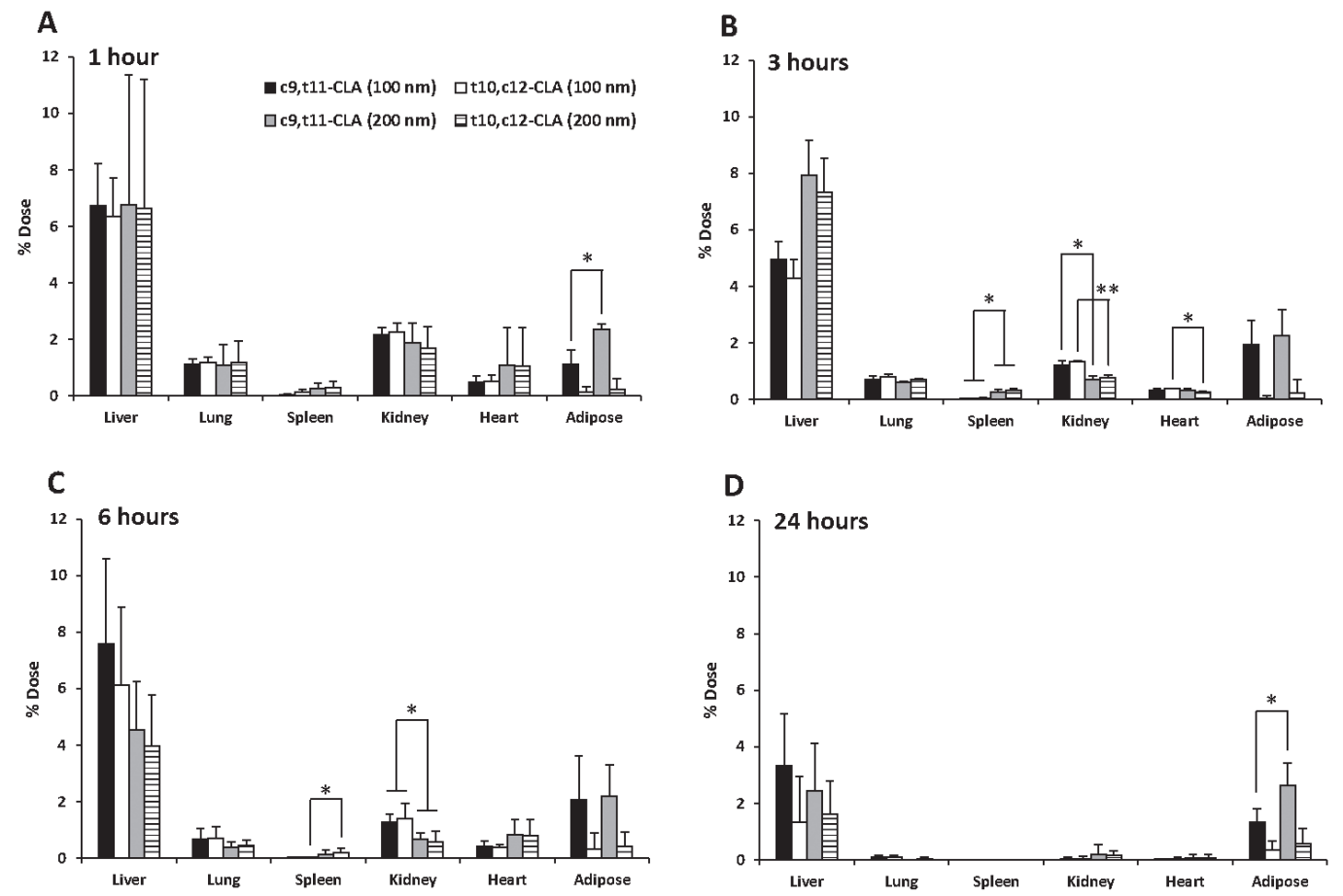

Fig. 3 Biodistribution of CLA in BALB/c mice. Mice were administered CLA-ME by intravenous injection. At indicated times, liver, lung, spleen, kidney, heart, and adipose tissues were collected and subjected to GC analysis. Data represent means \pm SEM for $3-4$ mice per group. Asterisks denote significant differences at $p<0.05(*)$ or $p<0.01$ $(* *)$.

CLA-ME was significantly more abundant than $100 \mathrm{~nm}$ CLA-ME in the spleen at $3 \mathrm{~h}$ and $6 \mathrm{~h}$, whereas the opposite was true for the kidney at the same time points. Both CLA-ME isomers, $c 9, t 11$-CLA and $t 10, c 12$-CLA, maintained the same ratio in all examined tissues. Finally, CLA-ME could be hardly detected in the blood and other tissues at $24 \mathrm{~h}$ after injection (Figs. 2 and 3D).

\section{Discussion}

In the present study, we prepared CLA-ME using premix membrane emulsification and investigated their pharmacokinetics in mice. As shown in Fig. 1 and Table 1, we obtained ME with a narrow size distribution and, based on zeta potential, negatively charged particle surface. Zeta potential values define the stability of colloids in water and comprise five intervals: from 0 to $\pm 5 \mathrm{mV}$ (rapid coagulation or flocculation), from \pm 10 to $\pm 30 \mathrm{mV}$ (incipient instability), from \pm 30 to $\pm 40 \mathrm{mV}$ (moderate stability), from \pm 40 to $\pm 60 \mathrm{mV}$ (good stability), more than $\pm 61 \mathrm{mV}$ (excellent stability ${ }^{17}$. Although our zeta potential results indicated incipient instability, CLA-ME were confirmed to maintain their size for more than a year. Although the detailed molecular mechanism is not elucidated, we empirically observed that HCO-60 could contribute to the stability of emulsions. PDI values define the distribution of particle di- ameter ranges from 0 to 1 , with 0 being monodisperse and 1 being polydisperse. The values of both CLA-ME were below 0.1 , indicating their monodispersity.

In emulsifying CLA, we chose HCO-60 as a co-surfactant, because this compound was reported to prevent uptake of lipid ME by the reticuloendothelial system(RES) and thus prolong their retention in the blood ${ }^{18,19)}$. Given how rapidly $t 10, c 12$-CLA is metabolized, it is critical that the appropriate blood concentration of $\mathrm{ME}$ is maintained long enough to exploit the physiological benefits of CLA-ME. Blood CLA concentration measurements showed that $100 \mathrm{~nm}$ CLA-ME were more stable than $200 \mathrm{~nm}$ CLA-ME at $6 \mathrm{~h}$ post injection and maintained the same ratio of CLA isomers during the experiments (Fig. 2). Accordingly, small CLA-ME might best meet our objectives. CLA-ME tended to accumulate mainly in the liver, followed by lungs and kidney. Differences in liver accumulation at 3 and $6 \mathrm{~h}$ after administration indicated that large ME were trapped by the RES in the early period. It was previously reported that small size nanoparticles exhibited longer circulation times compared with larger ones, which were rapidly taken up by the liver ${ }^{20,21}$. Macrophages in the liver, spleen, and lungs, known as kupffer cells or alveolar macrophages, are involved in RES uptake ${ }^{22,23)}$. Although it is unclear whether these cells contribute to CLA-ME clearance, consistent with the results of other reports ${ }^{24)}$, we have shown a decreased presence of large CLA-ME in the liver and spleen. Both CLA-ME could be 
hardly detected in the body at $24 \mathrm{~h}$ (Figs. 2 and 3D), and there was no appreciable difference in their half-life. It was reported that $227 \mathrm{~nm}$ melittin-loaded perfluorocarbon nanoparticle synthesized as an oil-in-water emulsion had a half-life of approximately $6.5 \mathrm{~h}^{25)}$, similar to that of $200 \mathrm{~nm}$ CLA-ME ( $6 \mathrm{~h})$. Moreover, we observed that only $c 9$, t11-CLA accumulated in adipose tissue, suggesting that CLA-ME might not be useful for targeting this tissue.

\section{Conclusion}

In summary, we prepared CLA-ME with a narrowly distributed size range, using CLA triglyceride and HCO-60 as a co-surfactant. Animal experiments revealed that, compared with 200 nm CLA-ME, 100 nm CLA-ME might be able to avoid uptake by the RES and benefit from a prolonged circulation time during the early period. However, given that CLA concentration in vivo could not be maintained at $24 \mathrm{~h}$, intravenous administration should take into account CLA-ME half-life, and future studies should try to improve it.

\section{References}

1) Tanaka, K. Occurrence of conjugated linoleic acid in ruminant products and its physiological functions. Anim. Sci. J. 76, 291-303(2005).

2) Park, H.S.; Ryu, J.H.; Ha, Y.L.; Park, J.H. Dietary conjugated linoleic acid (CLA) induces apoptosis of colonic mucosa in 1,2-dimethylhydrazine-treated rats: a possible mechanism of the anticarcinogenic effect by CLA. Br. J. Nutr. 86, 549-555(2001).

3) Kritchevsky, D.; Tepper, S.A.; Wright, S.; Tso, P.; Czarnecki, S.K. Influence of conjugated linoleic acid (CLA) on establishment and progression of atherosclerosis in rabbits. J. Am. Coll. Nutr. 19, 472S-477S (2000).

4) Park, Y.; Albright, K.J.; Liu, W.; Storkson, J.M.; Cook, M.E.; Pariza, M.W. Effect of conjugated linoleic acid on body composition in mice. Lipids 32, 853-858(1997).

5) Ryder, J.W.; Portocarrero, C.P.; Song, X.M.; Cui, L.; Yu, M.; Combatsiaris, T.; Galuska, D.; Bauman, D.E.; Barbano, D.M.; Charron, M.J.; Zierath, J.R.; Houseknecht, K.L. Isomer-specific antidiabetic properties of conjugated linoleic acid. Improved glucose tolerance, skeletal muscle insulin action, and UCP-2 gene expression. Diabetes 50, 1149-1157 (2001).

6) Inoue, N.; Nagao, K.; Hirata, J.; Wang, Y.M.; Yanagata, T. Conjugated linoleic acid prevents the development of essential hypertension in spontaneously hypertensive rats. Biochem. Biophys. Res. Commun. 323, 679-684 (2004).

7) Yamasaki, M.; Miyamoto, Y.; Chujo, H.; Nishiyama, K.;
Tachibana, H.; Yamada, K. Trans10, cis12-conjugated linoleic acid induces mitochondria-related apoptosis and lysosomal destabilization in rat hepatoma cells. Biochim. Biophys. Acta-Mol. Cell. Biol. Lipids 1735, 176-184(2005).

8) Yamasaki, M.; Chujo, H.; Koga, Y.; Rikimaru, T.; Shimada, M.; Sugimachi, K.; Tachibana, H.; Yamada, K. Potent cytotoxic effect of trans-10, cis12 isomer of conjugated linoleic acid on rat hepatoma dRLh-84 cells. Cancer Lett. 188, 171-180 (2002).

9) Białek, A.; Tokarz, A.; Dudek, A.; Kazimierska, W.; Bielecki, W. Influence of diet enriched with conjugated linoleic acids on their distribution in tissues of rats with DMBA induced tumors. Lipids Health Dis. 9, $126(2010)$.

10) Martin, J.C.; Gregoire, S.; Siess, M.H.; Genty, M.; Chardigny, J.M.; Berdeaux, O.; Juaneda, P.; Sebedio, J.L. Effects of conjugated linoleic acid isomers on lipid metabolising enzymes in male rats. Lipids 35, 91-98 (2000).

11) Yamasaki, M.; Ikeda, A.; Hirao, A.; Tanaka, Y.; Miyazaki, Y.; Rikimaru, T.; Shimada, M.; Sugimachi, K.; Tachibana, H.; Yamada, K. Effect of dietary conjugated linoleic acid on the in vivo growth of rat hepatoma dRLh-84. Nutr. Cancer 40, 140-148(2001).

12) Matsumura, Y.; Maeda, H. A new concept for macromolecular therapeutics in cancer chemotherapy: mechanism of tumoritropic accumulation of proteins and the antitumor agent SMANCS. Cancer Res. 46, 6387-6392(1986).

13) Maeda, H. The enhanced permeability and retention (EPR) effect in tumor vasculature: the key role of tumor-selective macromolecular drug targeting. Adv. Enzyme. Regul. 41,189-207(2001).

14) Kim, D.; Park, J.H.; Kweon, D.J.; Han, G.D. Bioavailability of nanoemulsified conjugated linoleic acid for an antiobesity effect. Int. J. Nanomed. 8, 451-459 (2013).

15) Nakashima, T.; Shimizu, M.; Kukizaki, K. Particle control of emulsion by membrane emulsification and its applications. Adv. Drug Deliv. Rev. 45, 47 (2000).

16) Yamasaki, M.; Motonaga, C.; Yokoyama, M.; Ikezaki, A.; Kakihara, T.; Hayasegawa, R.; Yamasaki, K.; Sakono, M.; Sakakibara, Y.; Suiko, M.; Nishiyama, K. Induction of apoptotic cell death in HL-60 cells by jacaranda seed oil derived fatty acids. J. Oleo Sci. 62, 925-932 (2013).

17) Duan, J.; Wang, J.; Guo, T.; Gregory, J. Zeta potentials and sizes of aluminum salt precipitates - Effect of anions and organics and implications for coagulation mechanisms. J. Water Pro. Eng. 4, 224-232 (2014).

18) Kurihara, A.; Shibayama, Y.; Mizota, A.; Yasuna, A.; Ikeda, M.; Sasagawa, K.; Kobayashi, T.; Hisaoka. M. Lipid emulsions of palmitoylrhizoxin: effects of com- 
position on lipolysis and biodistribution. Biopharm. Drug Dispos. 17, 331-342 (1996).

19) Lin, S.Y.; Wu, W.H.; Lui, W.Y. In vitro release, pharmacokinetic and tissue distribution studies of doxorubicin hydrochloride (Adriamycin $\mathrm{HCl}$ ) encapsulated in lipiodolized w/o emulsions and w/o/w multiple emulsions. Pharmazie 47, 439-443(1992).

20) Takino, T.; Konishi, K.; Takakura, Y.; Hashida, M. Long circulating emulsion carrier systems for highly lipophilic drugs. Biol. Pharm. Bull. 17, 121-125 (1994).

21) Yadav, K.S.; Chuttani, K.; Mishra, A.K.; Sawant, K.K. Effect of size on the biodistribution and blood clearance of etoposide-loaded PLGA nanoparticles. PDA J. Pharm. Sci. Technol. 65, 131-139(2011).

22) Litzinger, D.C.; Buiting, A.M.; van Rooijen, N.; Huang, L. Effect of liposome size on the circulation time and intraorgan distribution of amphipathic poly (ethyleneglycol)-containing liposomes. Biochim. Biophys. Acta 1190, 99-107 (1994)

23) Herant, M.; Heinrich. V.; Dembo, M. Mechanics of neutrophil phagocytosis: experiments and quantitative models. J. Cell Sci. 119, 1903-1913(2006).

24) Abra, R.M.; Hunt, C.A. Liposome disposition in vivo. III. Dose and vesicle-size effects. Biochim. Biophys. Acta 666, 493-503 (1981).

25) Soman, N.R.; Baldwin, S.L.; Hu, G.; Marsh, J.N.; Lanza, G.M.; Heuser, J.E.; Arbeit, J.M.; Wickline, S.A.; Schlesinger, P.H. Molecularly targeted nanocarriers deliver the cytolytic peptide melittin specifically to tumor cells in mice, reducing tumor growth. J. Clin. Invest. 119, 2830-2842 (2009). 